EPSC Abstracts

Vol. 15, EPSC2021-802, 2021

https://doi.org/10.5194/epsc2021-802

European Planetary Science Congress 2021

(c) Author(s) 2021. This work is distributed under

the Creative Commons Attribution 4.0 License.

\title{
Distribution of RV- and transiting exoplanets by masses and orbital periods taking into account observational selection
}

\author{
Vladislava Ananyeva ${ }^{1}$, Alexander Tavrov ${ }^{1}$, Oleg Korablev ${ }^{1}$, and Jean-Loup Bertaux ${ }^{2}$ \\ ${ }^{1}$ Space Research Institute of the Russian Academy of Sciences (IKI), Lab 501, Moscow, Russian Federation (a-lada@yandex.ru) \\ ${ }^{2}$ LATMOS/IPSL/CNRS/UVSQ, 78280, Guyancourt, France
}

More than $95 \%$ of the known exoplanets were discovered by the transit- and radial velocity techniques. However, the observed distributions of planets by their masses and by their orbital periods are significantly distorted by numerous observational selections, different for these techniques and different surveys.

We found and studied the de-biased statistical distributions of exoplanets by masses and by orbital periods for three groups of exoplanets: I. transiting planets discovered by Kepler ST, whose masses were measured by the follow-up radial velocity technique, II. transiting planets discovered by ground-based surveys (SuperWASP, HATNet, NGTS, XO, KELT, etc.), III. planets discovered by the radial velocity technique. The synthetic projective-mass distribution of RV planets obeys the piecewise power law with the breakpoints at $\sim 0.14 \mathrm{MJ}$ and $\sim 1.7 \mathrm{MJ}$. The distribution of RV-planets with $\mathrm{m}=(0.011-0.087) \mathrm{MJ}$ (or (3.5-28)ME) accurately obeys the power law with an exponent of -2 , $\mathrm{dN} / \mathrm{dm} \square \mathrm{m}^{\wedge}-2$. The distribution of RV planets with $\mathrm{m}=(0.21-1.7) \mathrm{MJ}$ follows the power law with an exponent ranging from -0.7 to $-0.8, \mathrm{dN} / \mathrm{dm} \square \mathrm{m}^{\wedge}(-0.7 \ldots-0.8)$. The distribution of RV planets with $\mathrm{m}$ $=(1.7-13) \mathrm{MJ}$ is fitted by a power law with an exponent ranging from -1.7 to $-2.0, \mathrm{dN} / \mathrm{dm} \square$ $\mathrm{m}^{\wedge}(-1.7 \ldots-2.0)$. In general, the synthetic projective-mass distribution of RV planets well agrees with the predictions of the population synthesis theory (Mordasini, 2018) and includes more detailed features to be discussed.

The exoplanets distribution by periods generally follows a power law with an exponent of -0.75 and indicates a predominant (averaged) structuring of the planetary systems.

De-biased mass distribution of RV-planets with orbital periods of 2-58 days is well consistent with a similar distribution of the Kepler planets. The mass distribution of the transit exoplanets detected by ground-based surveys is consistent with the similar distribution of RV planets with periods of 1-20 days. 